\title{
Numerical Analysis of Spectral Properties of Coupled Oscillator Schrödinger Operators I. Single and Double Well Anharmonic Oscillators
}

\author{
By D. Isaacson*, E. L. Isaacson**, D. Marchesin*** and P. J. Paes-Leme**
}

\begin{abstract}
We describe several methods for computing many eigenvalues and eigenfunctions of a single anharmonic oscillator Schrödinger operator whose potential may have one or two minima. One of the methods requires the solution of an ill-conditioned generalized eigenvalue problem. This method has the virtue of using a bounded amount of work to achieve a given accuracy in both the single and double well regions. We give rigorous bounds, and we prove that the approximations converge faster than any inverse power of the size of the matrices needed to compute them.

We present the results of our computations for the $g: \phi^{4}:_{1}$ theory. These results indicate that the methods actually converge exponentially fast. We conjecture why this is so.
\end{abstract}

1. Introduction. In statistical mechanics, quantum chemistry, and quantum field theory [1], numerical techniques for solving eigenvalue problems in a large or an infinite number of variables are required. A procedure is described in [1] for dealing with the case of a large system of coupled anharmonic oscillators.

This paper provides the initial step of the procedure. That is, an algorithm is devised to find efficiently and accurately the eigenvalues and eigenfunctions of one anharmonic oscillator for the case of either a single or a double well region. We describe simple algorithms including a method which seems to be applicable to multi-well problems in general. This method involves an overdetermined basis and allows a careful fitting of the levels associated with each well. The multi-well method has the desirable feature that the amount of work needed to compute the first $N$ eigenvalues and eigenfunctions to a given accuracy is uniformly bounded in the entire parameter space. The algorithms we describe are convergent and yield rigorous bounds on the accuracy of the approximate eigenvalues and eigenfunctions.

We remark that there is a large literature on the numerical study of anharmonic oscillators. One of the earliest articles to present bounds for the single well problem is [2]. We refer the reader to the recent article [3] for more references.

In Section 2 we formulate the problem, and in Sections 3-6 we describe the algorithms. Section 6 contains an error analysis of the algorithms including derivations of the rigorous bounds mentioned above. In Section 7 we describe the results obtained from applying our algorithms to the $: \phi^{4}:_{1}$ theory.

Received November 30, 1979; revised January 16, 1981.

1980 Mathematics Subject Classification. Primary 65N25; Secondary 81-04.

* Supported by the National Science Foundation under Grant No. MCS-77-03568.

** Supported in part by the National Science Foundation under Grant No. PHY-78-08066.

*** Supported in part by NASA Grant NSG-5034. 
We prove in Appendix 5 that the approximate eigenvalues and eigenvectors converge faster than any negative power of the size of the matrices used to compute them. We conjecture that the convergence is actually exponentially fast.

2. Formulation. In order to illustrate the algorithms, we consider the Hamiltonian operator

$$
H \equiv \frac{1}{2}\left(-\frac{d^{2}}{d x^{2}}+c x^{2}+d x^{4}+e\right)=\frac{1}{2}\left(-\frac{d^{2}}{d x^{2}}+V(x)\right)
$$

where $d$ is any positive number, and $c, e$ are aribtrary real numbers. We use the following notation. The eigenvalues of $H$ are $E_{j}, j=0,1,2, \ldots$, with the corresponding eigenfunctions $\psi_{j}, j=0,1,2, \ldots$ Here, $E_{0}<E_{1}<E_{2}<\ldots$, and

$$
H \psi_{j}=E_{j} \psi_{j}
$$

We remark that the operator $H$ commutes with the unitary operator

$$
U \psi(x) \equiv \psi(-x)
$$

Therefore, the subspaces of $L_{2}(\mathbf{R})$ of even function $\left(\mathcal{H}_{e}\right)$ and odd functions $\left(\mathcal{H}_{o}\right)$ are invariant under $H$, and computing the lowest $N$ eigenvalues in each of these subspaces provides us with the lowest $2 N$ eigenvalues of $H$.

In each algorithm we choose a basis ${ }^{\dagger}\left\{\phi_{j}\right\}=\left\{\phi_{j}(x)\right\}_{j=0}^{\infty}$ of $L_{2}(\mathbf{R})$ (actually, we choose bases $\left\{\phi_{j}^{e}\right\}$ of $\mathcal{K}_{e}$ and $\left\{\phi_{j}^{o}\right\}$ of $H_{o}$ ) and observe that for some coefficients $\left\{v_{j, k}^{(n)}\right\}$

$$
\psi_{j}=\lim _{n \rightarrow \infty} \sum_{k=0}^{n} v_{j, k}^{(n)} \phi_{k}(x) .
$$

Substituting (2.4) in (2.2) and taking the inner product with $\phi_{l}, l=0,1,2, \ldots$, yields

$$
\begin{aligned}
\left\langle H^{*} \phi_{l}, \lim _{n \rightarrow \infty} \sum_{k=0}^{n} v_{j, k}^{(n)} \phi_{k}\right\rangle & =E_{j}\left\langle\phi_{l}, \lim _{n \rightarrow \infty} \sum_{k=0}^{n} v_{j, k}^{(n)} \phi_{k}\right\rangle, \\
\lim _{n \rightarrow \infty} \sum_{k=0}^{n}\left\langle H \phi_{l}, \phi_{k}\right\rangle v_{j, k}^{(n)} & =\lim _{n \rightarrow \infty} E_{j} \sum_{k=0}^{n}\left\langle\phi_{l}, \phi_{k}\right\rangle v_{j, k}^{(n)} .
\end{aligned}
$$

This leads naturally to the following procedure: Compute numerically the eigenvalues $E_{j}^{(n)}$ and eigenvectors $v_{j}^{(n)}$ of the generalized eigenvalue problem

$$
H^{(n)} v_{j}^{(n)}=E_{j}^{(n)} S^{(n)} v_{j}^{(n)}
$$

where $H^{(n)}$ and $S^{(n)}$ are the $(n+1) \times(n+1)$ matrices with components

$$
\begin{array}{rlrl}
H_{l, k} & \equiv\left\langle\phi_{l}, H \phi_{k}\right\rangle, & l, k=0, \ldots, n, \\
S_{l, k} \equiv\left\langle\phi_{l}, \phi_{k}\right\rangle, & l, k=0, \ldots, n,
\end{array}
$$

and $v_{j}^{(n)}$ is the $(n+1)$ component vector

$$
v_{j}^{(n)} \equiv\left(v_{j, 0}^{(n)}, \ldots, v_{j, n}^{(n)}\right)^{T}
$$

Then $E_{j}^{(n)}$ is an approximation of $E_{j}$, and

$$
\psi_{j}^{(n)}(x) \equiv \sum_{k=0}^{n} v_{j, k}^{(n)} \phi_{k}(x)
$$

\footnotetext{
${ }^{\dagger}$ By a basis of a space we mean a subset whose linear span is dense in that space.
} 
is an approximation of $\psi_{j}(x)$. It follows from (A5.3) in Appendix 5 that the $E_{j}^{(n)}$ are Rayleigh-Ritz upper bounds for the $E_{j}$.

The algorithms, therefore, consist of the following steps:

Step 1: Choosing a basis $\left\{\phi_{j}\right\}_{j=0}^{\infty}$.

Step 2: Computing the matrix elments (2.6), (2.7).

Step 3: Solving the matrix eigenvalue problems (2.5).

Step 4: Performing an error analysis.

The last step is optional since it is used only to determine how the algorithms are converging. The analysis consists of computing (for increasing sequences of $l$ 's and n's)

$$
\begin{aligned}
\Delta E_{j}(n, l) & \equiv\left|E_{j}^{(l)}-E_{j}^{(n)}\right|, \\
\Delta \psi_{j}(n, l) & \equiv\left\|\psi_{j}^{(l)}-\psi_{j}^{(n)}\right\|,
\end{aligned}
$$

and the "operator residuals"

$$
r_{j}(n) \equiv\left\|H \psi_{j}^{(n)}-E_{j}^{(n)} \psi_{j}^{(n)}\right\| .
$$

The information above can be used to derive bounds on the actual errors $\mid E_{j}^{(n)}-$ $E_{j} \mid,\left\|\psi_{j}^{(n)}-\psi_{j}\right\| \cdot$. (See Section 6.)

We now describe each of the four steps in more detail.

3. Step 1: Choice of a Basis. In order to keep the matrix eigenvalue problem (2.4) small in size, the basis functions $\phi_{j}$ (actually $\phi_{j}^{e}$ and $\phi_{j}^{o}$ ) should be good approximations for the eigenfunctions $\psi_{j}$. Two other practical requirements are: (i) the matrix elements (2.6), (2.7) should be easy to compute, and (ii) the eigenvalue problem (2.5) should be easy to solve numerically.

We observe that for $c>0$ and $d=0$ the eigenfunctions $\psi_{j}$ of $H$ are just the Hermite functions $\Omega_{j}(x)$ given by

$$
\begin{gathered}
\Omega_{0}(x) \equiv\left(\frac{\alpha}{\pi}\right)^{1 / 4} e^{-\frac{\alpha}{2} x^{2}}, \\
\Omega_{j}(x) \equiv\left(2^{j} j !\right)^{-1 / 2}\left[\alpha^{1 / 2} x-\alpha^{-1 / 2} d / d x\right]^{j} \Omega_{0}(x), \quad j=1,2,3, \ldots,
\end{gathered}
$$

where $\alpha$ is chosen to minimize the Rayleigh quotient

$$
R(\alpha) \equiv\left\langle\Omega_{0}, H \Omega_{0}\right\rangle /\left\langle\Omega_{0}, \Omega_{0}\right\rangle .
$$

We remark that $\alpha$ can be computed in closed form.

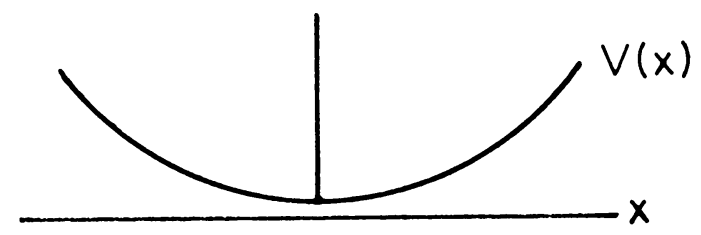

FIGURE 1

$V(x)$ vs $x$ in the single well region

For $c>0$ and $d \neq 0$, the potential has approximately the same shape near its minimum $(x=0)$ as it does for $d=0$. (See Figure 1.) In this region of the parameter space, we choose $\left\{\phi_{j}^{e}\right\}$ to be the even Hermite functions and $\left\{\phi_{j}^{o}\right\}$ to be 
the odd Hermite functions. (We refer to this region of parameter space as the "Gaussian region", and we call each basis above a "Gaussian basis.")

For $c<0$ and $d$ small, the potential has two widely separated minima (see Figure 2), and the Gaussian basis yields poor approximations for the eigenfunctions. In this region of the parameter space, the eigenfunctions are approximated well by linear combinations of Hermite functions translated to the minima of the potential [4], [5].

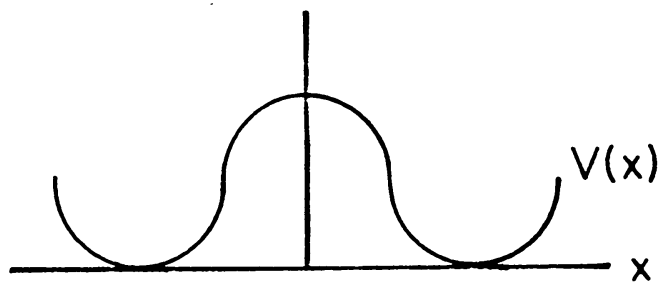

FIGURE 2

$V(x)$ vs $x$ in the double well region

Therefore, in this region we use the bases defined by

$$
\begin{gathered}
\phi_{j}^{e} \equiv \frac{\Omega_{j}^{-}+\Omega_{j}^{+}}{2^{1 / 2}}, \quad j=0,1,2, \ldots, \\
\phi_{j}^{o} \equiv \frac{\Omega_{j}^{-}-\Omega_{j}^{+}}{2^{1 / 2}}, \quad j=0,1,2, \ldots,
\end{gathered}
$$

where

$$
\Omega_{j}^{-}(x) \equiv \Omega_{j}\left(\alpha\left(x-x_{0}\right)\right), \quad \Omega_{j}^{+}(x) \equiv \Omega_{j}\left(-\alpha\left(x+x_{0}\right)\right) .
$$

The constants $\alpha$ and $x_{0}$ are determined so as to minimize the Rayleigh quotient

$$
R_{1}\left(\alpha, x_{0}\right) \equiv\left\langle\phi_{0}^{e}, H \phi_{0}^{e}\right\rangle /\left\langle\phi_{0}^{e}, \phi_{0}^{e}\right\rangle \text {. }
$$

(We refer to this region of parameter space as the "Ising region", and we call each of the bases (3.4) an "Ising basis" because of the relation between the double well problem and the one-dimensional Ising model [5], [6].) It is shown in Appendix 1 that $\left\{\phi_{j}^{e}\right\}$ and $\left\{\phi_{j}^{o}\right\}$ are nonminimal bases ${ }^{t+}$ of $\mathcal{H}_{e}$ and $\mathcal{K}_{o}$, respectively.

4. Step 2: Computation of the Matrix Elements. In this section we describe the methods for computing the matrix elements (2.6), (2.7). We restrict our attention to the even subspace $\mathcal{H}_{e}$ since the formulas in $\mathcal{H}_{o}$ are similar.

For the Gaussian basis the matrix, $S^{(n)}$ is the identity since the Hermite functions are orthonormal. Also, the matrix elements $H_{j, k}$ can be computed easily using recursion relations for the even (or odd) Hermite functions yielding a five-diagonal band matrix. (An explicit formula is given in Appendix 6.)

To compute $S^{(n)}$ for the Ising basis (3.4e), we have

$$
S_{j, k}=\left\langle\phi_{j}^{e}, \phi_{k}^{e}\right\rangle=\frac{1}{2}\left\langle\Omega_{j}^{-}+\Omega_{j}^{+}, \Omega_{k}^{-}+\Omega_{k}^{+}\right\rangle=\delta_{j, k}+\left\langle\Omega_{j}^{+}, \Omega_{k}^{-}\right\rangle .
$$

\footnotetext{
\# By a nonminimal basis we mean a basis which has a proper subset that is also a basis [15].
} 
We have used the identities

$$
U \Omega_{j}^{-}=\Omega_{j}^{+}, \quad U \Omega_{k}^{+}=\Omega_{k}^{-},
$$

and the fact that $U$ given by (2.3) is unitary. The matrix elements $U_{j, k} \equiv\left\langle\Omega_{j}^{+}, \Omega_{k}^{-}\right\rangle$ (which are a representation of $U$ with respect to either orthonormal basis $\left\{\Omega_{j}^{+}\right\}$or $\left.\left\{\Omega_{j}^{-}\right\}\right)$are Gaussian integrals which can be computed accurately by Gaussian quadrature [7].

For $H_{j, k}$ we have

$$
\begin{aligned}
H_{j, k} & =\left\langle\phi_{j}^{e}, H \phi_{k}^{e}\right\rangle=\frac{1}{2}\left\langle\Omega_{j}^{-}+\Omega_{j}^{+}, H\left(\Omega_{k}^{-}+\Omega_{k}^{+}\right)\right\rangle \\
& =\left\langle\Omega_{j}^{+}, H \Omega_{k}^{+}\right\rangle+\left\langle\Omega_{j}^{+}, H \Omega_{k}^{-}\right\rangle .
\end{aligned}
$$

We have used (4.2) again and the fact that $H$ and $U$ commute.

The infinite matrix $H^{+}$, with elements $H_{j, k}^{+} \equiv\left\langle\Omega_{j}^{+}, H \Omega_{k}^{+}\right\rangle$, is a nine-diagonal band matrix because of the form of $H$ and the recursion relations for the Hermite functions. To compute $\left\langle\Omega_{j}^{+}, H \Omega_{k}^{-}\right\rangle$, we expand $\Omega_{k}^{-}$with respect to the orthonormal basis $\left\{\Omega_{l}^{+}\right\}$obtaining

$$
\begin{aligned}
\left\langle\Omega_{j}^{+}, H \Omega_{k}^{-}\right\rangle & =\sum_{l=0}^{\infty}\left\langle\Omega_{j}^{+}, H \Omega_{l}^{+}\right\rangle\left\langle\Omega_{l}^{+}, \Omega_{k}^{-}\right\rangle=\sum_{l=0}^{\infty} H_{j, l}^{+} U_{l, k} \\
& =\sum_{l=j-4}^{j+4} H_{j, l}^{+} U_{l, k} .
\end{aligned}
$$

The last equality follows since $H^{+}$is nine-diagonal. We may therefore write (4.1), (4.3) as the infinite matrix identities ${ }^{+1}$

$$
\mathrm{S}=\mathrm{I}+\mathrm{U} ; \quad \mathrm{H}=\mathrm{H}^{+}+\mathrm{H}^{+} \mathrm{U}=\mathrm{H}^{+} \mathrm{S} .
$$

In the odd subspace $\mathcal{H}_{o}$ the formulas become

$$
S=I-U ; \quad H=H^{+} S .
$$

5. Step 3: Solution of the Eigenvalue Problem (2.5). In this section we present methods for solving

$$
H^{(n)} v_{j}^{(n)}=E_{j}^{(n)} S^{(n)} v_{j}^{(n)}
$$

in both the Gaussian and Ising regions. Again, we restrict attention to the even subspace $\mathcal{K}_{e}$.

In the Gaussian region (5.1) becomes

$$
H^{(n)} v^{(n)}=\lambda^{(n)} v^{(n)}
$$

where $H^{(n)}$ is five-diagonal. We solve (5.2) in a conventional manner using Eispack [8]: First, $H^{(n)}$ is reduced to tridiagonal form; then the eigenvalues of the resulting system are found by the $Q R$ algorithm; lastly, the eigenvectors are determined by inverse iteration.

The problem (5.1) is more difficult in the Ising region. Since $S^{(n)}$ is the Gram matrix of the basis $\left\{\phi_{j}^{e}\right\}_{j=0}^{n}$ and since, as shown in Appendix 1, the bases $\left\{\phi_{j}^{e}\right\}$ and $\left\{\phi_{j}^{0}\right\}$ are nonminimal, the matrix $S^{(n)}$ is nearly singular. Therefore, (5.1) is illconditioned.

\footnotetext{
Itt We use the same symbol to denote an operator and a corresponding infinite matrix. The context should determine which meaning is desired.
} 
We now present two methods which overcome this defect. The second method is preferable, however, since it is two to five times faster than the first method.

The first method derives from the following observations (see Appendix 2): $H^{(n)}$ and $S^{(n)}$ have nearly the same null space, and they almost commute. Therefore, we first diagonalize $S^{(n)}$ :

$$
S^{(n)}=Q D Q^{T}
$$

where $Q$ is the orthogonal matrix of eigenvectors of $S^{(n)}$, and $D$ is the diagonal matrix of eigenvalues. We then restrict $H^{(n)}$ and $S^{(n)}$ to the subspace spanned by the eigenvectors of $S^{(n)}$ corresponding to those eigenvalues larger than a preset tolerance. That is, we consider the smaller problem

$$
\tilde{H}^{(n)} \tilde{v}_{j}^{(n)}=E_{j}^{(n)} \tilde{D} \tilde{v}_{j}^{(n)},
$$

where

$$
\tilde{H}^{(n)}=\tilde{Q}^{T} H^{(n)} \tilde{Q}, \quad \tilde{D}=\tilde{Q}^{T} S^{(n)} \tilde{Q},
$$

and $\tilde{Q}$ is obtained from $Q$ by deleting the eigenvectors corresponding to small eigenvalues. $v_{j}^{(n)}$ is obtained from $\tilde{v}_{j}^{(n)}$ by

$$
v_{j}^{(n)}=\tilde{Q} \tilde{v}_{j}^{(n)}
$$

Since $\tilde{D}$ is a diagonal positive matrix, (5.3) can be solved by standard methods.

The choice of the tolerance mentioned above is delicate. Too large a tolerance degrades the accuracy of the solution because useful information is neglected. Too small a tolerance introduces spurious unstable eigenvalues. Using the CDC 6600 computer (with fourteen decimal digits of accuracy), we found satisfactory values of the tolerance to range from $10^{-8}$ to $10^{-10}$.

In the second method we eliminate the (nearly) dependent basis elements from $\left\{\phi_{j}^{e}\right\}_{j=0}^{n}$ using a Cholesky decomposition with maximal pivoting (see Appendix 3). The matrix $\tilde{S}^{(n)}$ which results is strictly positive. Therefore, after replacing $S^{(n)}$ with $\tilde{S}^{(n)}, H^{(n)}$ with $\tilde{H}^{(n)}$, and $v_{j}^{(n)}$ with $\tilde{v}_{j}^{(n)}$ using the independent basis elements, we can use the following standard procedure to solve (5.1): Make the change of variables $v=D^{1 / 2} L^{T} \tilde{v}_{j}^{(n)}$, where $\tilde{S}^{(n)}=L D L^{T}$ is the Cholesky decomposition. Then solve the resulting symmetric eigenvalue problem

$$
\left(L D^{1 / 2}\right)^{-1} \tilde{H}^{(n)}\left(D^{1 / 2} L^{T}\right)^{-1} v=E v .
$$

6. Step 4: Error Analysis. In order to determine how the algorithms are converging, we compute the following quantities:

$$
\begin{aligned}
\Delta E_{j}(n, l) & \equiv\left|E_{j}^{(l)}-E_{j}^{(n)}\right|, \\
\Delta \psi_{j}(n, l) & \equiv\left\|\psi_{j}^{(l)}-\psi_{j}^{(n)}\right\|,
\end{aligned}
$$

and the "operator residuals"

$$
r_{j}(n) \equiv\left\|H \psi_{j}^{(n)}-E_{j}^{(n)} \psi_{j}^{(n)}\right\|
$$

The roles that $\Delta E_{j}$ and $\Delta \psi_{j}$ play in determining convergence are clear. Tables are presented in Section 7. However, the $r_{j}(n)$ are more important since they allow us to compute rigorous bounds on the errors in the approximate eigenvalues and eigenvectors. 
Let $E$ denote the eigenvalue of $H$ closest to $E_{j}^{(n)}$, let $\psi$ be the corresponding eigenvector, and let $\Delta$ denote the distance between $E_{j}^{(n)}$ and the next closest eigenvalue of $H$. Then [9]-[12]

$$
\begin{gathered}
\left|E_{j}^{(n)}-E\right| \leqslant r_{j}(n), \quad\left|E_{j}^{(n)}-E\right| \leqslant r_{j}(n)^{2} / \Delta, \\
\left\|\psi_{j}^{(n)}-\psi\right\| \leqslant \frac{r_{j}(n)}{\Delta}\left(1+\left(\frac{r_{j}(n)}{\Delta}\right)^{2}\right)^{1 / 2} .
\end{gathered}
$$

We use these inequalities to obtain upper and lower bounds for $E$ by computing a rigorous lower bound for $\Delta$. Let $\delta_{j} \equiv \min \left\{\left|E_{j}^{(n)}-E_{j-1}^{(n)}\right|,\left|E_{j}^{(n)}-E_{j+1}^{(n)}\right|\right\}$ and $\tilde{r}_{j}(n)$ be either $r_{j-1}(n)$ or $r_{j+1}(n)$ according to whether $E_{j}^{(n)}$ is closer to $E_{j-1}^{(n)}$ or $E_{j+1}^{(n)}$. Then, for $n$ sufficiently large, $\Delta \geqslant \delta_{j}-\tilde{r}_{j}(n)$, and consequently

$$
\left|E_{j}^{(n)}-E_{j}\right|<r_{j}(n)^{2} /\left(\delta_{j}-\tilde{r}_{j}(n)\right) \text {. }
$$

We now prove the lower bound for $\Delta$ in the case when $E_{j}^{(n)}$ is closer to $E_{j+1}^{(n)}$ than to $E_{j-1}^{(n)}$. From Appendix 5 we can choose $n$ sufficiently large so that

$$
E_{j-1} \leqslant E_{j-1}^{(n)} \leqslant E_{j} \leqslant E_{j}^{(n)} \leqslant E_{j+1} \leqslant E_{j+1}^{(n)} \text {. }
$$

In this case $E_{j}^{(n)}$ is closer to $E_{j+1}$ than to $E_{j-1}$. Thus

$$
\Delta=E_{j+1}-E_{j}^{(n)}=E_{j+1}^{(n)}-E_{j}^{(n)}-\left(E_{j+1}^{(n)}-E_{j+1}\right) .
$$

From the definition of $\delta_{j}$ and $\tilde{r}_{j}(n)$ we have

$$
\Delta \geqslant \delta_{j}-r_{j+1}(n)=\delta_{j}-\tilde{r}_{j}(n) .
$$

The case where $E_{j}^{(n)}$ is closer to $E_{j-1}^{(n)}$ than to $E_{j+1}^{(n)}$ is similar.

The gap $\Delta$ may be estimated accurately by $\delta_{j}$ when $r_{j}(n)$ and $\tilde{r}_{j}(n)$ are much smaller than $\delta_{j}$. Typical values of $r_{j}(n)$ and $r_{j}(n)^{2} / \delta_{j}$ are given in Section 7 .

Calculation of the vector differences (6.2) is straightforward: if $n>l$ and we write $\psi_{j}^{(l)}-\psi_{j}^{(n)}=\sum_{k=0}^{n} w_{k} \phi_{k}^{e}$, then

$$
\left\|\psi_{j}^{(l)}-\psi_{j}^{(n)}\right\|^{2}=\sum_{k=0}^{n} \sum_{m=0}^{n}\left\langle\phi_{k}^{e}, \phi_{m}^{e}\right\rangle w_{k} w_{m}=w^{T} S^{(n)} w,
$$

where $w=\left(w_{0}, \ldots, w_{n}\right)^{T}$.

Calculation of the operator residuals is more complicated. In Appendix 4 we derive formulas for computing these in each basis.

7. Application to $: \phi^{4}:_{1}$. The : $\phi^{4}:_{1}$ theory is the study of the anharmonic oscillator operator

$$
H=H(g) \equiv(1 / 2)\left[-d^{2} / d x^{2}+(1-3 g) x^{2}+g x^{4}+c_{g}\right],
$$

where $0<g<\infty$, and $c_{g}$ is a constant chosen so that the lowest eigenvalue of $H(g)$ is 0 . We note that as $g$ goes from 0 to $\infty$ the potential changes its shape from having one to having two minima as illustrated in Figures 1 and 2.

As in the text we denote the eigenvalues and eigenfunctions of $H(g)$ by $E_{j}=E_{j}(g)$ and $\psi_{j}=\psi_{j}(g)$. When $g=0$ we have [13] $E_{j}(0)=j$, and $\psi_{j}(0)$ are just the Hermite functions. As $g \rightarrow \infty$ [2], [3], [4], the eigenvalues become degenerate in pairs, i.e.,

$$
E_{2 j}(g) \sim E_{2 j+1}(g) \sim(6 g)^{1 / 2} j \text { for } j=0,1,2, \ldots
$$


and the eigenfunctions approach an "Ising basis". In fact the precise rate of asymptotic eigenvalue degeneracy as $g \rightarrow \infty$ has been established [14].

We computed the first twenty eigenvalues and eigenfunctions of $H(g)$. Their behavior as functions of $g$ are displayed in Figures 4, 5 and 6.

Numerical values of $\Delta E_{j}(n, n+10)$ and $\Delta \psi_{j}(n, n+10)$ (defined by (6.1), (6.2)) for the Gaussian basis are given in Table 1. Values for the Ising basis are given in Table 2. They seem to show that $E_{j}^{(n)}$ and $\psi_{j}^{(n)}$ are converging with increasing $n$. In fact Figure 3 suggests that $E_{j}^{(n)}$ is approaching its limit exponentially fast. In Appendix 5 we give a simple proof that $E_{j}^{(n)}$ and $\psi_{j}^{(n)}$ converge to $E_{j}$ and $\psi_{j}$ faster than any power of $n^{-1}$.

Tables 1 and 2 include the operator residuals $r_{j}$ and $r_{j}^{2} / \delta_{j}$. We remark that the "computed" residuals provide rigorous bounds on the approximate eigenvalues and eigenvectors only if infinite precision arithmetic is used. We do not discuss here the effects of rounding in the computation of rigorous bounds; however, we point out that when $r_{j}^{2} / \delta_{j} \approx 10^{-23}$ and $E_{j}^{(n)}$ has been computed using only 14 digits it is highly unlikely that $\left|E_{j}-E_{j}^{(n)}\right| \approx O\left(10^{-23}\right)$.

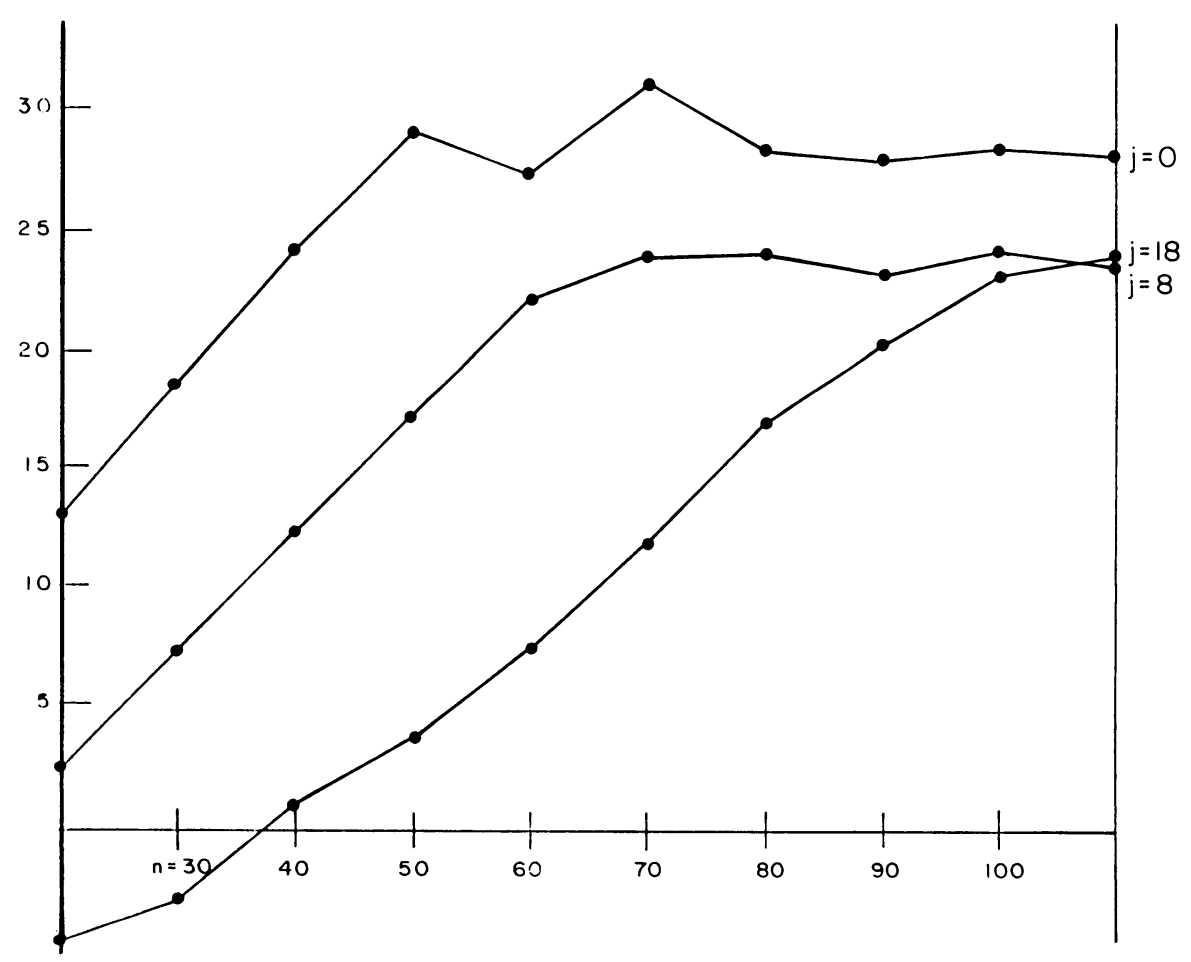

Figure 3

$-\ln \left[E_{j}^{(n)}-E_{j}^{(n+10)}\right]$ (Gaussian basis)

(The curves become flat when rounding prevents $E_{j}^{(n)}$ from improving) 


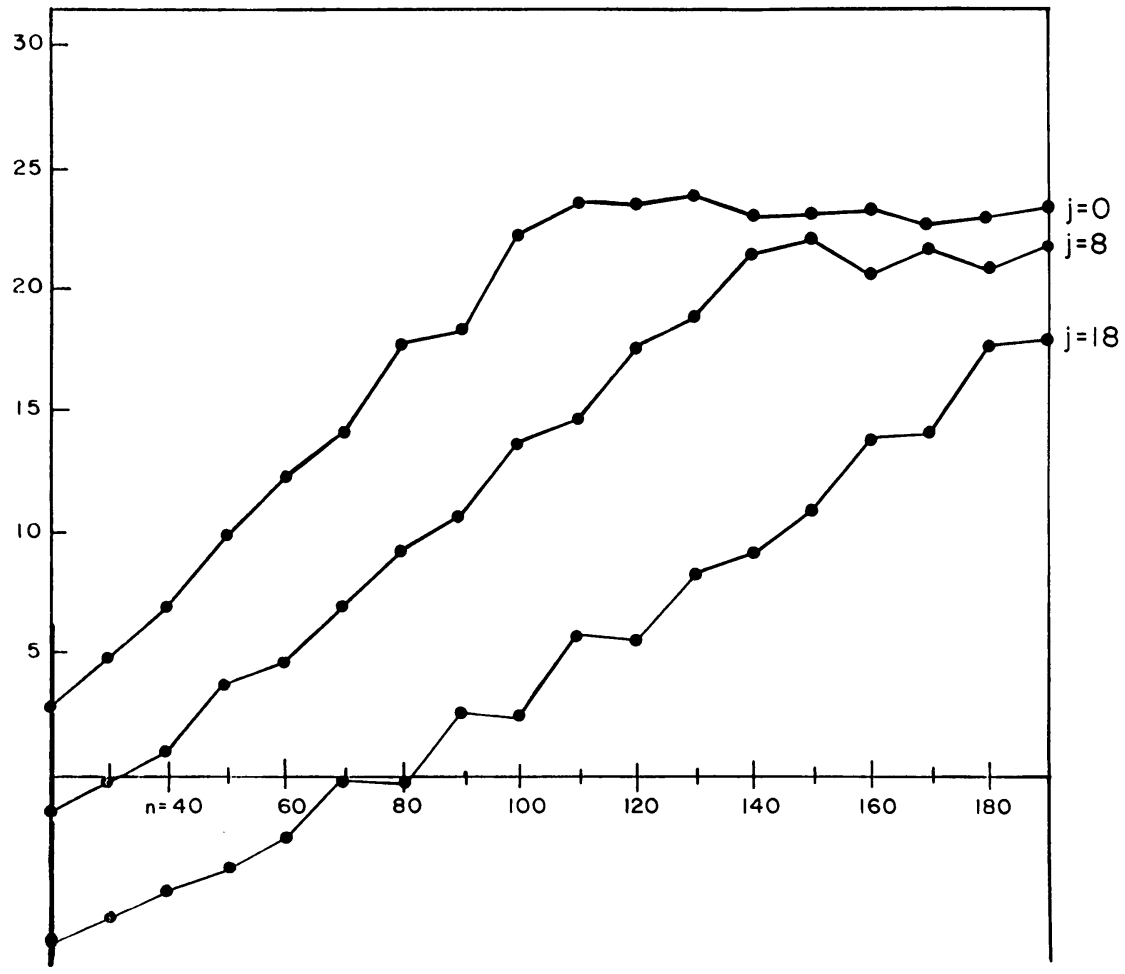

Figure 4. $E_{j}(g) v s g$

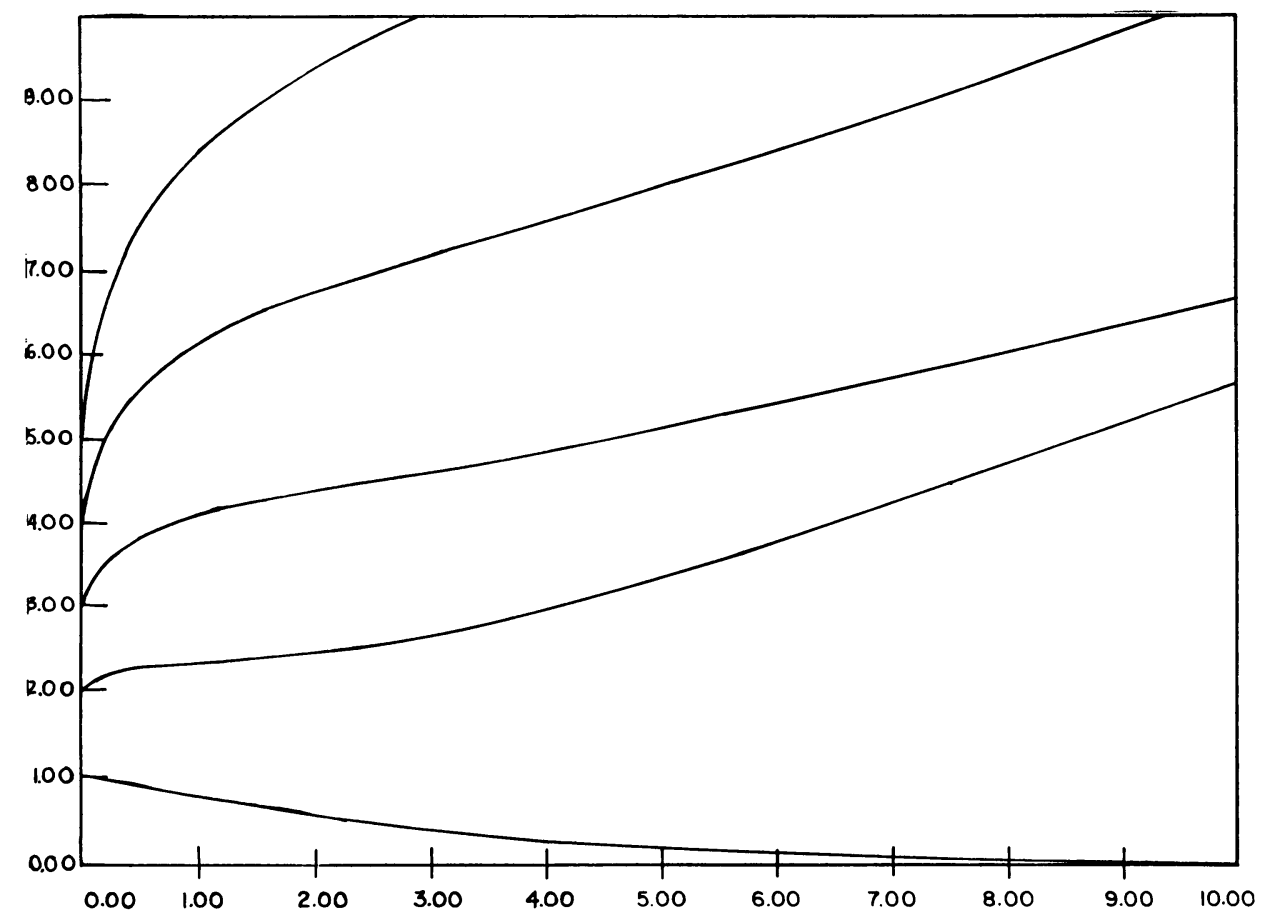

Figure 5. $E_{j}(g)$ vs $g$ 


\begin{tabular}{|c|c|c|c|c|}
\hline$i_{i}^{\infty}$ & $\stackrel{\infty}{\sim}$ & 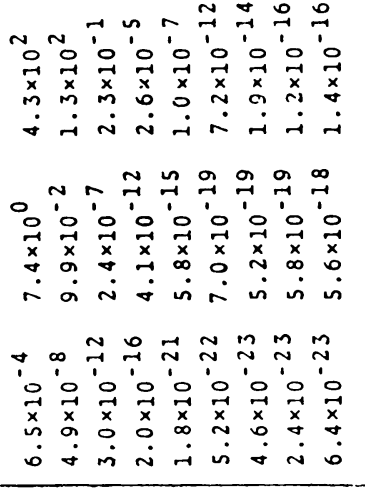 & $\stackrel{\infty}{\rightarrow}$ & 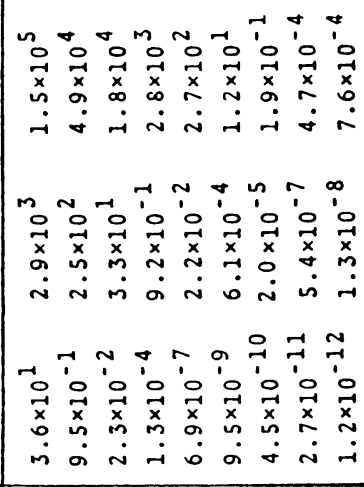 \\
\hline$\dot{m}$ & $\stackrel{\infty}{-1}$ & 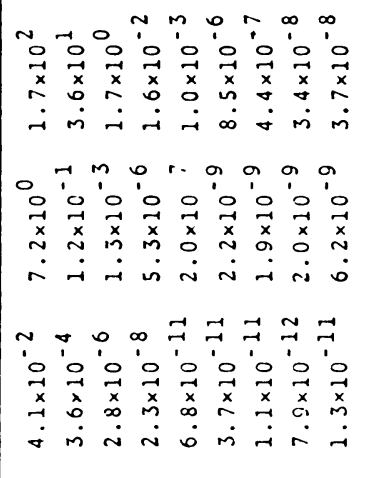 & $\stackrel{\infty}{-1}$ & 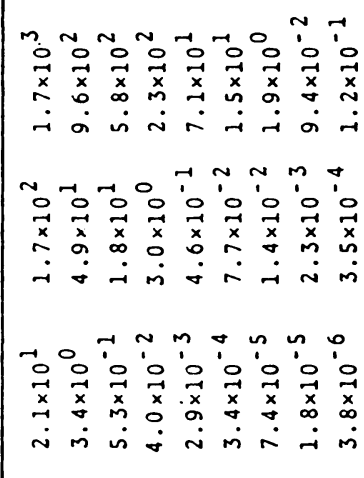 \\
\hline$\dot{\vec{a}}$ & 0 & 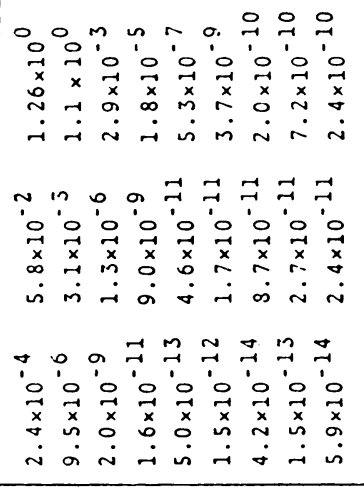 & $\stackrel{\infty}{\sim}$ & 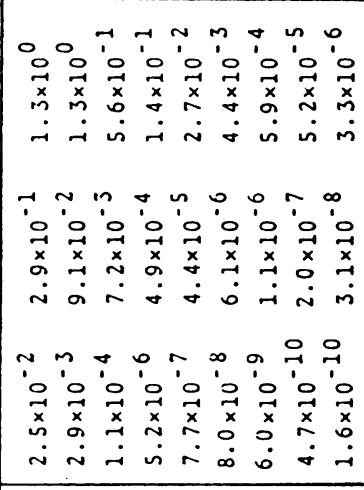 \\
\hline$\dot{y}_{j}$ & $\stackrel{\infty}{\sim}$ & 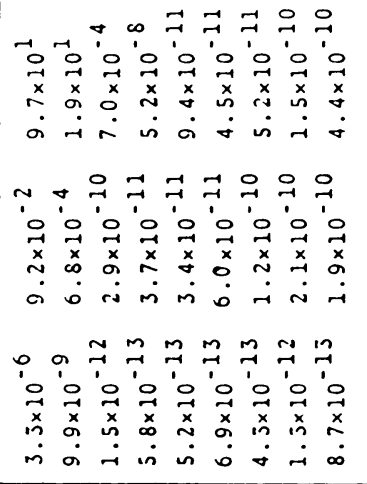 & $\stackrel{\infty}{\sim}$ & 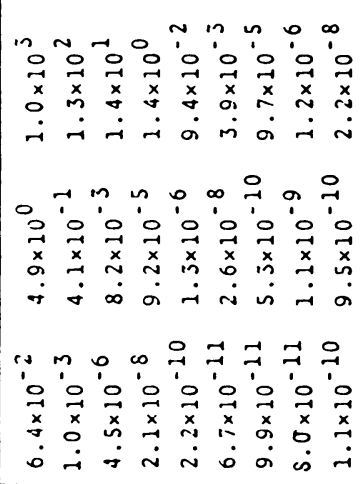 \\
\hline$\because$ & 工 & 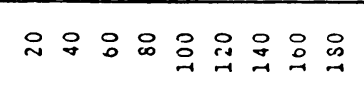 & $4=$ & 尺 \\
\hline
\end{tabular}




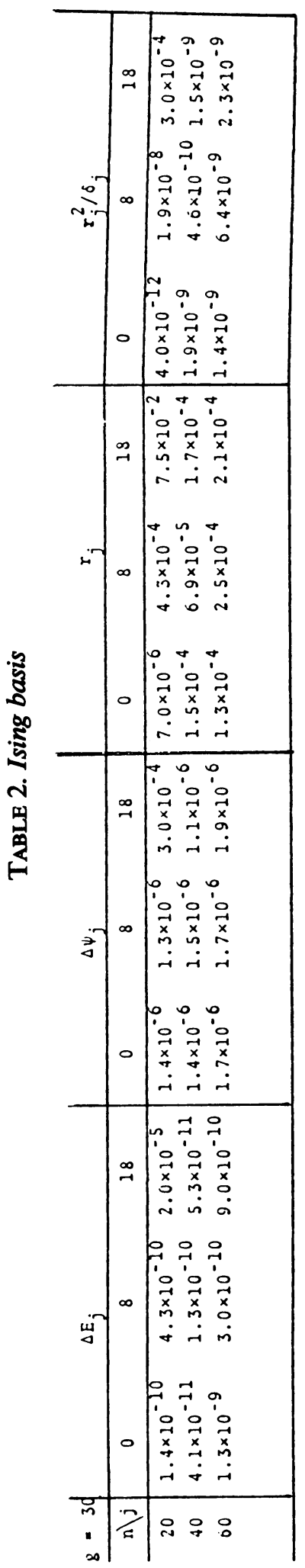




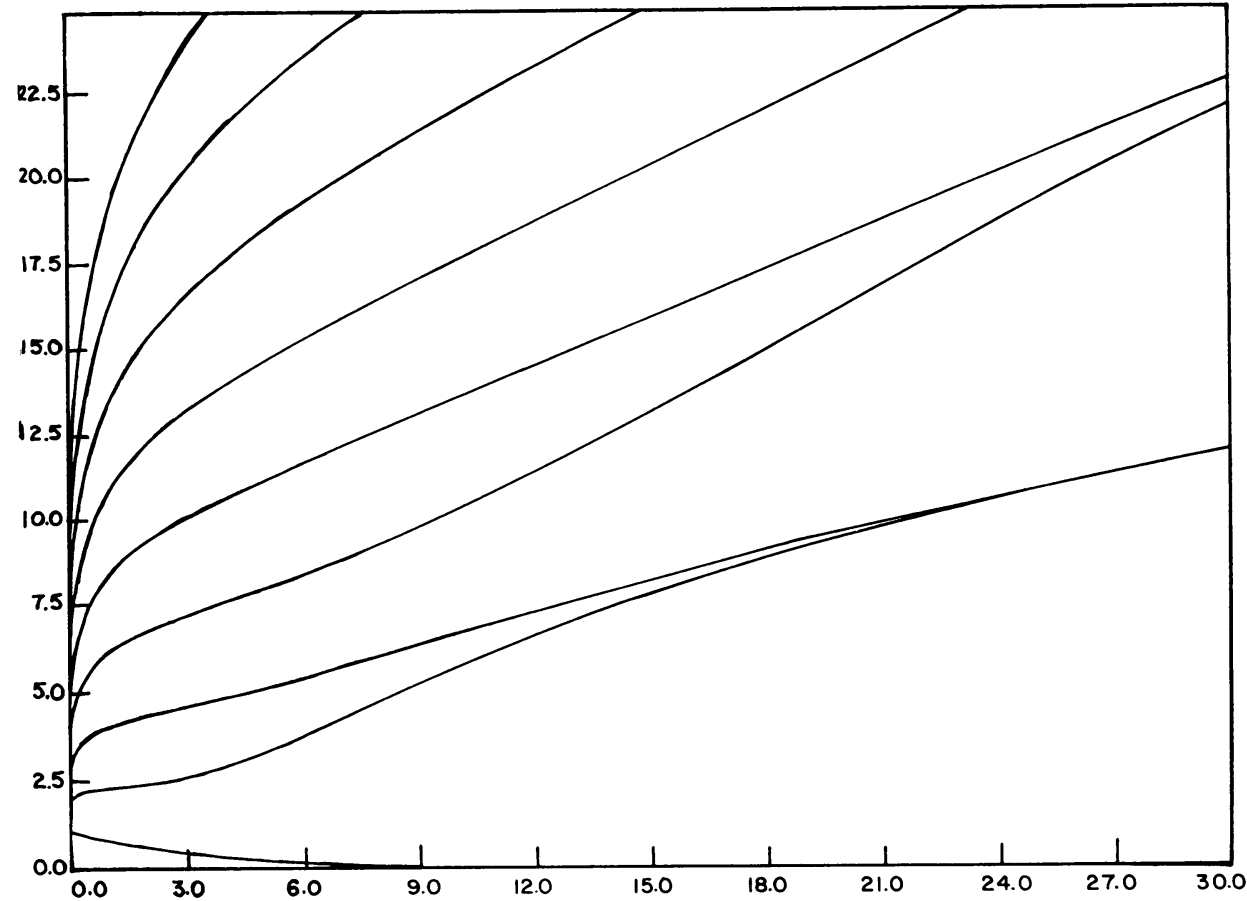

FIGURE 6. $E_{j}(g)$ vs $g$

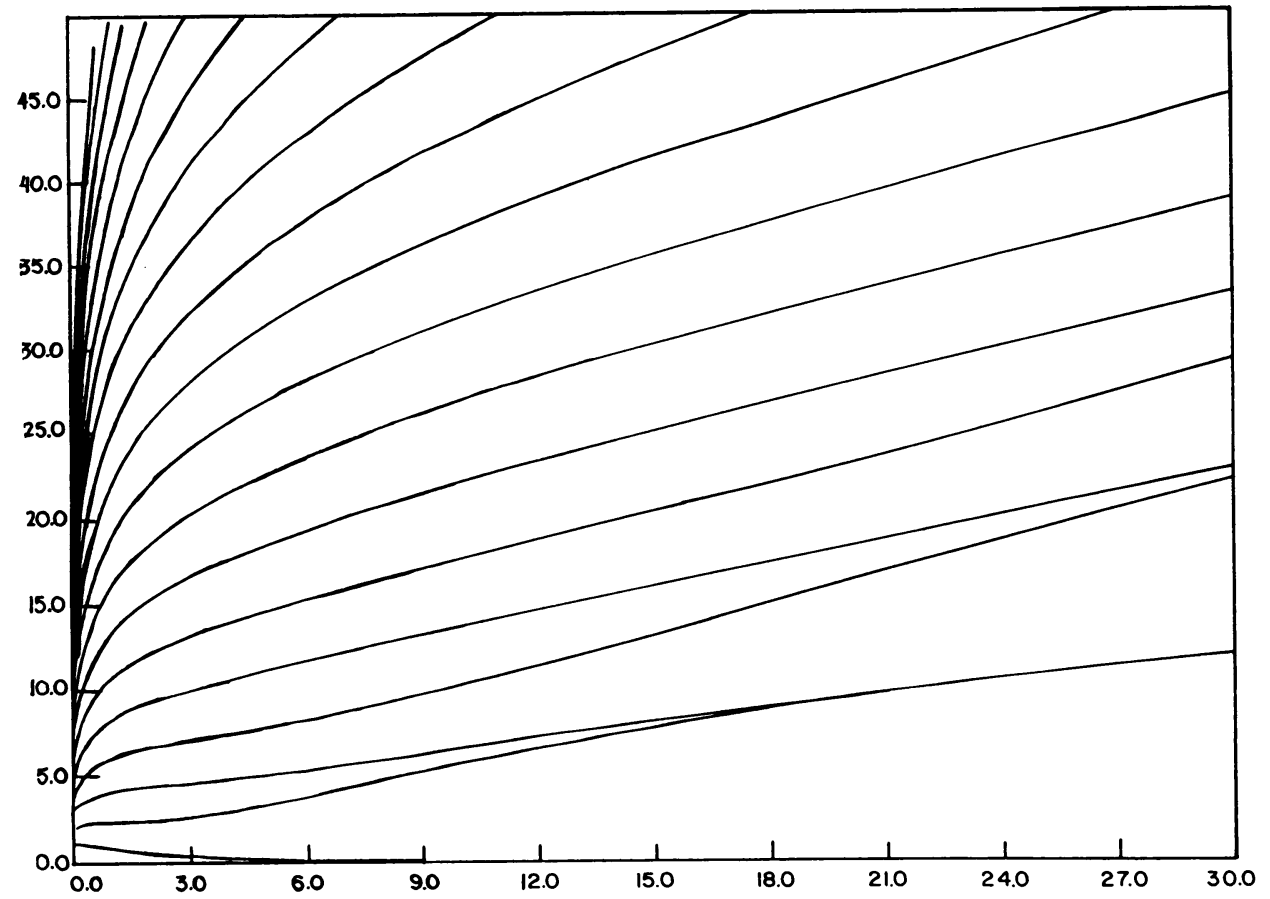

FIGURE 7. $E_{j}(g)$ vs $g$ 
Appendix 1: Nonminimality of the Ising Basis. In this appendix we show that the even Ising basis $(3.4 e)$ is a nonminimal basis for the subspace $\mathcal{H}_{e}$ of even square integrable functions. In fact, we prove that the subset $\left\{\phi_{2 j}^{e}\right\}_{j=0}^{\infty}$ is a basis that also is nonminimal. Similar results hold in $\mathcal{H}_{o}$ with the odd Ising basis (3.4o).

THEOREM A1. Let $\left\{\Omega_{j}\right\}_{j=0}^{\infty}$ be any orthonormal basis of $L_{2}(\mathbf{R})$ with $\Omega_{2 j}$ even and $\Omega_{2 j+1}$ odd. Define

$$
\Omega_{j}^{+}(x) \equiv \Omega_{j}\left(x+x_{0}\right), \quad \phi_{j}^{e}(x) \equiv 2^{-1 / 2}\left(\Omega_{j}^{+}(x)+\Omega_{j}^{+}(-x)\right) .
$$

Then the span of $\left\{\phi_{2 j}^{e}\right\}_{j=0}^{\infty}$ is dense in the subspace $\mathcal{H}_{e}$ of even functions of $L_{2}$.

Proof. Define the operator $S: \mathcal{F}_{e}^{x_{0}} \rightarrow \mathcal{K}_{e}$ by

$$
(S \psi)(x) \equiv \psi(x)+\psi(-x)
$$

where $\mathcal{F}_{e}^{x_{0}} \equiv\left\{\psi(x) \in L_{2}(\mathbf{R}): \psi\left(x+x_{0}\right)=\psi\left(-x+x_{0}\right)\right\}$ is the space of even functions about $x_{0}$. Since $\phi_{2 j}^{e}=2^{-1 / 2} S \Omega_{2 j}^{+},\left\{\Omega_{2 j}^{+}\right\}$is an orthonormal basis of $\mathcal{F}_{e}^{x_{0}}$, and $S$ is bounded, it suffices to show the range of $S$ is dense in $\mathcal{K}_{e}$.

So let $\chi \in \mathcal{H}_{e}$ be such that $\langle\chi, S \psi\rangle=0$ for all $\psi \in \mathcal{H}_{e}^{x_{0}}$. We will show that $\chi$ is zero.

Since $\chi \in \mathcal{H}_{e}$, a change of variable gives $0=\langle\chi, S \psi\rangle=2\langle\chi, \psi\rangle$ for all $\psi \in$ $\mathcal{H}_{e}^{x_{0}}$. Therefore, $\chi$ is odd about $\chi_{0}$. Since $\chi$ is also even about the origin, it follows by successive reflections that any such $\chi$ with finite norm is zero.

TheOrEM A2. With the notation of Theorem A1, if $\hat{\Omega}_{0}$ is positive, then $\left\{\phi_{2 j}^{e}\right\}_{j=0}^{\infty}$ is a nonminimal basis of $\mathcal{H}_{e}$. ( (denotes Fourier transform.)

We remark that the restriction on $\hat{\Omega}_{0}$ can be relaxed considerably.

Sketch of Proof. We have shown already that $\left\{\phi_{2 j}^{e}\right\}$ is a basis. To prove nonminimality, we show $\phi_{0}^{e} \in \operatorname{span}\left\{\phi_{2 j}^{e}\right\}_{j=1}^{\infty}$; i.e., $\left\|\phi_{0}^{e}-\sum_{n=1}^{N} a_{n}^{N} \phi_{2 n}^{e}\right\|$ can be made small by an appropriate choice of the constants. In fact, we will determine constants $b_{0}^{N}, \ldots, b_{N}^{N}$ so that $\left\|\sum_{n=0}^{N} b_{n}^{N} \phi_{2 n}^{e}\right\|$ is small, but $b_{0}^{N}$ is bounded away from zero.

A simple calculation shows

$$
\hat{\phi}_{2 n}^{e}(\xi)=2^{1 / 2} \cos \xi x_{0} \hat{\Omega}_{2 n}(\xi)
$$

Therefore, by the Plancherel theorem,

$$
\left\|\sum_{n=0}^{N} b_{n}^{N} \phi_{2 n}^{e}\right\|=\left\|\sum_{n=0}^{N} b_{n}^{N} \hat{\phi}_{2 n}^{e}\right\|=2^{1 / 2}\left\|\cos \xi x_{0} \sum_{n=0}^{N} b_{n}^{N} \hat{\Omega}_{2 n}\right\| .
$$

Now, take a function $\hat{f}(\xi)$ which is a sum of approximate delta functions at two symmetric zeros of $\cos \xi x_{0}$, say $\pm \pi / 2 x_{0}$. Then $\left\|\cos \xi x_{0} \hat{f}(\xi)\right\|$ is small.

Since the Fourier transform is unitary on $\mathcal{H}_{e},\left\{\hat{\Omega}_{2 j}\right\}_{j=0}^{\infty}$ is an orthonormal basis. Expanding $\hat{f}$ as $\hat{f}=\sum_{n=0}^{\infty} b_{n} \hat{\Omega}_{2 n}$ and then truncating yields the desired coefficients. Clearly, (A3.1) is small, and $b_{0}=\left\langle\hat{f}, \hat{\Omega}_{0}\right\rangle \approx \hat{\Omega}_{0}\left(\pi / 2 x_{0}\right)+\hat{\Omega}_{0}\left(-\pi / 2 x_{0}\right)$ is bounded away from zero.

Appendix 2. Properties of the Generalized Eigenvalue Problem. We describe properties of the generalized eigenvalue problem which motivate the first method explained in Section 4. 
From (4.5e), and a similar computation,

$$
H=H^{+} S=S H^{+},
$$

where $S$ is a matrix representation of a bounded operator and $H^{+}$is a matrix representation of an unbounded positive definite operator. (We ignore questions about domains in the following and assume the matrices in question are restricted to operating on rapidly decreasing sequences.)

It follows from (A2.1) that $H$ and $S$ commute and (since $H^{+}$is positive definite) that they have the same null space.

$S$ has a large null space. In fact, if $\chi \in \mathcal{S}$ is an odd function, then its components $\left\{v_{l}\right\}$ with respect to the basis $\left\{\Omega_{l}^{-}\right\}$are a rapidly decreasing null sequence for $S$ because

$$
\begin{aligned}
\sum_{l=0}^{\infty} S_{j, l} v_{l} & =\sum_{l=0}^{\infty}\left\langle\phi_{j}^{e}, \phi_{l}^{e}\right\rangle v_{l}=2^{-1} \sum_{l=0}^{\infty}\left\langle\Omega_{j}^{+}+\Omega_{j}^{-}, \Omega_{l}^{+}+\Omega_{l}^{-}\right\rangle v_{l} \\
& =\sum_{l=0}^{\infty}\left\langle\Omega_{j}^{+}+\Omega_{j}^{-}, \Omega_{l}^{-}\right\rangle v_{l}=2^{1 / 2}\left\langle\phi_{j}^{e}, \sum_{l=0}^{\infty} v_{l} \Omega_{l}^{-}\right\rangle \\
& =2^{1 / 2}\left\langle\phi_{j}^{e}, \chi\right\rangle=0 .
\end{aligned}
$$

We observe that the finite matrices $S^{(n)}$ and $H^{(n)}$ do not commute and do not have zero eigenvalues. They weakly commute in the sense that any fixed ( $n$ independent) element of $\left[H^{(n)}, S^{(n)}\right]$ tends to zero as $n$ tends to infinity, and they have approximately a common "spurious" space of eigenvectors with very small eigenvalues.

Appendix 3. The Cholesky Decomposition With Maximal Pivoting. The purpose of this method is to find a permutation $\left\{\phi_{j_{k}}\right\}_{k=0}^{n}$ of the original basis $\left\{\phi_{j}\right\}_{j=0}^{n}$ so that the "most independent" elements are first. Thus, the "most dependent" elements are last and can be eliminated easily from the original basis.

The Cholesky decomposition

$$
S=L D L^{T}
$$

of the Gram matrix $S=S^{(n)}$ of the original basis is particularly well suited for this purpose. For, the diagonal elements of $D$ satisfy

$$
D_{k, k}=\operatorname{det} S^{(k)} / \operatorname{det} S^{(k-1)}
$$

and the right side is the square of the distance from $\phi_{k}$ to the hyperplane spanned by $\phi_{0}, \ldots, \phi_{k-1}$. We are thus led to the following pivoting strategy in the calculation of (A3.1):

(a) Choose $\phi_{j_{0}}$ to have maximum norm.

(b) Assuming $\phi_{j_{0}}, \ldots, \phi_{j_{k-1}}$ have been chosen, choose $\phi_{j_{k}}$ so that the new $D_{k, k}$ is maximal.

Therefore, $\phi_{j_{k}}$ is farther from the span of $\phi_{j_{0}}, \ldots, \phi_{j_{k-1}}$ than any of the remaining basis elements.

To eliminate the "most dependent" elements, we omit those elements for which $D_{k, k}$ is less than a prescribed tolerance. (The remarks on the tolerance in Section 5 apply here as well.) Since the diagonal elements decrease in this method, we simply stop the procedure when the first small diagonal element is found. 
We remark that this algorithm is applied to a finite set for several reasons. First, for an infinite basis there is no guarantee that there is any element $\phi_{j_{k}}$ which is "most independent" of the previous ones. Furthermore, if a sequence $\left\{\phi_{j_{k}}\right\}$ is found, it will not necessarily span the original space. However, minor modifications can be made to overcome both of these obstacles.

Appendix 4. Calculation of Operator Residuals. We derive explicit formulas for the operator residuals (6.3) in terms of quantities previously calculated in Section 4. Throughout this appendix we let $P_{n}$ denote orthogonal projection onto $\operatorname{span}\left\{\phi_{j}\right\}_{j=0}^{n}$ for both the Ising basis and the Gaussian basis.

Formulas in the Ising Region. Using the Ising basis, we get

$$
\begin{aligned}
r_{j}(n)^{2}= & \left\|\left(H-E_{j}^{(n)}\right) \psi_{j}^{(n)}\right\|^{2}=\left\langle\psi_{j}^{(n)}, H^{2} \psi_{j}^{(n)}\right\rangle \\
& -2 E_{j}^{(n)}\left\langle\psi_{j}^{(n)}, H \psi_{j}^{(n)}\right\rangle+E_{j}^{(n)^{2}}\left\langle\psi_{j}^{(n)}, \psi_{j}^{(n)}\right\rangle .
\end{aligned}
$$

However,

$$
\left\langle\psi_{j}^{(n)}, H \psi_{j}^{(n)}\right\rangle=\left\langle\psi_{j}^{(n)}, P_{n} H P_{n} \psi_{j}^{(n)}\right\rangle=E_{j}^{(n)}\left\langle\psi_{j}^{(n)}, \psi_{j}^{(n)}\right\rangle
$$

since $P_{n} \psi_{j}^{(n)}=\psi_{j}^{(n)}$ and $P_{n} H P_{n} \psi_{j}^{(n)}=E_{j}^{(n)} \psi_{j}^{(n)}$. Substituting (A4.2) in (A4.1) yields

$$
r_{j}(n)^{2}=\left\langle\psi_{j}^{(n)}, H^{2} \psi_{j}^{(n)}\right\rangle-E_{j}^{(n)^{2}}\left\langle\psi_{j}^{(n)}, \psi_{j}^{(n)}\right\rangle
$$

We now evaluate the first term without calculating the matrix elements of the operator $H^{2}$ directly. In $\mathcal{H}_{e}$ we have

$$
\begin{aligned}
\left\langle\psi_{j}^{(n)}, H^{2} \psi_{j}^{(n)}\right\rangle=\sum_{k, l=0}^{n} v_{j, k}^{(n)} v_{j, l}^{(n)}\left\langle\phi_{k}^{e}, H^{2} \phi_{l}^{e}\right\rangle, \\
\left\langle\phi_{k}^{e}, H^{2} \phi_{l}^{e}\right\rangle=2^{-1}\left\langle\Omega_{k}^{+}+\Omega_{k}^{-}, H^{2}\left(\Omega_{l}^{+}+\Omega_{l}^{-}\right)\right\rangle \\
=\left\langle\Omega_{k}^{+}, H^{2} \Omega_{l}^{+}\right\rangle+\left\langle\Omega_{k}^{+}, H^{2} \Omega_{l}^{-}\right\rangle .
\end{aligned}
$$

Now,

$$
\begin{aligned}
\left\langle\Omega_{k}^{+}, H^{2} \Omega_{l}^{+}\right\rangle & =\left\langle H \Omega_{k}^{+}, H \Omega_{l}^{+}\right\rangle=\left\langle H \Omega_{k}^{+}, \sum_{m=0}^{\infty}\left\langle\Omega_{m}^{+}, H \Omega_{l}^{+}\right\rangle \Omega_{m}^{+}\right\rangle \\
& =\sum_{m=0}^{\infty}\left\langle H \Omega_{k}^{+}, \Omega_{m}^{+}\right\rangle\left\langle\Omega_{m}^{+}, H \Omega_{l}^{+}\right\rangle \\
& =\sum_{m=M_{1}}^{M_{2}}\left\langle\Omega_{k}^{+}, H \Omega_{m}^{+}\right\rangle\left\langle\Omega_{m}^{+}, H \Omega_{l}^{+}\right\rangle,
\end{aligned}
$$

where $M_{2}=\min \{k, l\}+4, M_{1}=\max \{k, l, 4\}-4$. For the last term of (A4.5) we obtain, similarly,

$$
\left\langle\Omega_{k}^{+}, H^{2} \Omega_{l}^{-}\right\rangle=\sum_{m=k-8}^{k+8}\left\langle\Omega_{k}^{+}, H^{2} \Omega_{m}^{+}\right\rangle\left\langle\Omega_{m}^{+}, \Omega_{l}^{-}\right\rangle .
$$

Equations (A4.6), (A4.7) reduce the calculation of (A4.3) to that of the matrix elements of $\mathrm{H}^{+}$and $S$ which were determined in Section 4. We remark that it would not have been difficult to compute the matrix elements of $H^{2}$ directly, and for other operators this may be advisable. 
Formulas in the Gaussian Region. Letting $Q_{n} \equiv I-P_{n}$, we get

$$
\begin{aligned}
& \text { (A4.8) } \begin{aligned}
r_{j}(n)^{2} & =\left\|\left(H-E_{j}^{(n)}\right) \psi_{j}^{(n)}\right\|^{2}=\left\|\left(P_{n}+Q_{n}\right)\left(H-E_{j}^{(n)}\right)\left(P_{n}+Q_{n}\right) \psi_{j}^{(n)}\right\|^{2} \\
& =\left\|Q_{n} H \psi_{j}^{(n)}\right\|^{2},
\end{aligned} \\
& \text { since } P_{n}\left(H-E_{j}^{(n)}\right) \psi_{j}^{(n)}=0=Q_{n} \psi_{j}^{(n)} .
\end{aligned}
$$

Now write

$$
H=A+B,
$$

where $A$ is the Hermite operator with eigenfunctions $\left\{\Omega_{j}\right\}$ and $B$ is the remaining fourth-degree polynomial. It follows that $Q_{n}$ commutes with $A$ so that

$$
\begin{aligned}
r_{j}(n)^{2} & =\left\|Q_{n} B \psi_{j}^{(n)}\right\|^{2}=\left\|\left(I-P_{n}\right) B \psi_{j}^{(n)}\right\|^{2} \\
& =\left\langle\psi_{j}^{(n)}, B^{2} \psi_{j}^{(n)}\right\rangle-\left\langle\psi_{j}^{(n)}, B P_{n} B \psi_{j}^{(n)}\right\rangle .
\end{aligned}
$$

For the first term we have (writing $\psi_{j}^{(n)}=\sum_{k=0}^{n} v_{j, k}^{(n)} \Omega_{k}$ )

$$
\left\langle\psi_{j}^{(n)}, B^{2} \psi_{j}^{(n)}\right\rangle=\sum_{k, l=0}^{n}\left\langle\Omega_{k}, B^{2} \Omega_{l}\right\rangle v_{j, k}^{(n)} v_{j, l}^{(n)},
$$

and a calculation similar to (A4.6) yields

$$
\left\langle\Omega_{k}, B^{2} \Omega_{l}\right\rangle=\sum_{m=0}^{\infty}\left\langle\Omega_{k}, B \Omega_{m}\right\rangle\left\langle\Omega_{m}, B \Omega_{l}\right\rangle .
$$

Similarly, we obtain for the last term of (A4.10)

$$
\left\langle\psi_{j}^{(n)}, B P_{n} B \psi_{j}^{(n)}\right\rangle=\sum_{k, l=0}^{n}\left\langle\Omega_{k}, B P_{n} B \Omega_{l}\right\rangle v(n, k) v_{j, l}^{(n)}
$$

and

$$
\left\langle\Omega_{k}, B P_{n} B \Omega_{l}\right\rangle=\sum_{m=0}^{n}\left\langle\Omega_{k}, B \Omega_{m}\right\rangle\left\langle\Omega_{m}, B \Omega_{l}\right\rangle .
$$

Substituting (A4.11-14) into (A4.10) gives

$$
r_{j}(n)^{2}=\sum_{k, l=M}^{n}\left[\sum_{m=n+1}^{N} B_{m, k} B_{m, l}\right] v_{j, k}^{(n)} v_{j, l}^{(n)},
$$

where

(A4.16) $M \equiv \max \{0, n-3\}, \quad N \equiv \min \{k+4, l+4\}, \quad B_{m, k} \equiv\left\langle\Omega_{m}, B \Omega_{k}\right\rangle$.

We have used the fact that $\left\langle\Omega_{m}, B \Omega_{k}\right\rangle=0$ for $|m-k|>4$.

Therefore, the residuals in the Gaussian basis can be computed from (A4.15), (A4.16). The numbers $B_{m, k}$ are determined by simple recursion relations. (See formulas (A6.1) and (A6.3) in Appendix 6.)

Appendix 5. Convergence of the Eigenvalues and Eigenfunctions. For the Gaussian and Ising bases we have the following

THEOREM. Fix $j \geqslant 0$. Then for every $k>0$ there are constants $c_{1}$ and $c_{2}$ so that for all $n>n_{0}(j)$ (independent of $\left.k\right)$

$$
\begin{aligned}
& \left|E_{j}^{(n)}-E_{j}\right|<c_{1} n^{-k} \\
& \left\|\psi_{j}^{(n)}-\psi_{j}\right\|<c_{2} n^{-k}
\end{aligned}
$$


[We sketch a proof for the Gaussian basis. Various proofs of convergence of Rayleigh-Ritz-Galerkin methods may be found in [16]-[18]. A nice proof for the case of finite elements is in [19].]

Proof of (A5.1). Let $\left\{\Omega_{j}\right\}_{j=0}^{\infty}$ be the Hermite functions given by (3.1)-(3.3), and let $P_{n}$ denote orthogonal projection onto $\mathcal{F}^{(n)} \equiv \operatorname{span}\left\{\Omega_{j}\right\}_{j=0}^{n}$. From the minimax principle we have

$$
E_{j}\left\langle E_{j}^{(n)}=\min _{\mathscr{S C}_{j}^{(n)} \subset \mathcal{T}^{(n)}} \max _{\psi \in \mathcal{T C}^{(n)}} \frac{\langle\psi, H \psi\rangle}{\langle\psi, \psi\rangle},\right.
$$

where $\mathcal{H}_{j}^{(n)}$ denotes a $(j+1)$-dimensional subspace of $\mathcal{H}^{(n)}$. If $V_{j} \equiv \operatorname{span}\left\{\psi_{k}\right\}_{k=0}^{j}$ and $V_{j}^{(n)} \equiv \operatorname{span}\left\{P_{n} \psi_{k}\right\}_{k=0}^{j}$, then $V_{j}^{(n)}$ is $(j+1)$-dimensional for $n$ sufficiently large because the Gram matrix $\left[\left\langle P_{n} \psi_{l}, P_{n} \psi_{k}\right\rangle\right\}_{l, k=0}^{j}$ approaches the identity matrix as $n \rightarrow \infty$. Thus (A5.3) becomes

$$
\begin{aligned}
0 & \left\langle E_{j}^{(n)}-E_{j}\left\langle\max _{\left.\psi \in V V^{n}\right)} \frac{\langle\psi, H \psi\rangle}{\langle\psi, \psi\rangle}-E_{j}=\max _{\psi \in V\}^{(n)}} \frac{\langle\psi, H \psi\rangle}{\langle\psi, \psi\rangle}-\left\langle\psi, H \psi_{j}\right\rangle\right.\right. \\
& =\max _{\psi \in V_{j}} \frac{\left\langle P_{n} \psi, H P_{n} \psi\right\rangle}{\left\langle P_{n} \psi, P_{n} \psi\right\rangle}-\max _{\psi \in V_{j}} \frac{\langle\psi, H \psi\rangle}{\langle\psi, \psi\rangle} \\
& \left\langle\max _{\substack{\psi \in V_{j} \\
\|\psi\|=1}}\left[\frac{\left\langle P_{n} \psi, H P_{n} \psi\right\rangle}{\left\langle P_{n} \psi, P_{n} \psi\right\rangle}-\langle\psi, H \psi\rangle\right] .\right.
\end{aligned}
$$

Since $\left\|P_{n} \psi\right\| \leqslant 1$ and $\left\|P_{n} \psi\right\| \rightarrow 1$, we may write $\left\|P_{n} \psi\right\|^{-2}=1+\varepsilon_{n}$, where $\varepsilon_{n}>0$ and $\varepsilon_{n} \rightarrow 0$. From (A5.4) we get

$$
\text { (A5.5) } 0<E_{j}^{(n)}-E_{j} \leqslant \max _{\substack{\psi \in V_{j} \\\|\psi\|=1}}\left[\left\langle P_{n} \psi, H P_{n} \psi\right\rangle-\langle\psi, H \psi\rangle+\varepsilon_{n}\left\langle P_{n} \psi, H P_{n} \psi\right\rangle\right] \text {. }
$$

However,

$$
\begin{aligned}
\mid\left\langle P_{n} \psi, H P_{n} \psi\right\rangle-\langle\psi & H \psi\rangle \mid \\
& =\left|\left\langle\left(P_{n} \psi-\psi\right), H P_{n} \psi\right\rangle+\left\langle\psi, H\left(P_{n} \psi-\psi\right)\right\rangle\right| \\
& <\left\|H^{1 / 2}\left(P_{n} \psi-\psi\right)\right\|\left\|H^{1 / 2} P_{n} \psi\right\|+\|H \psi\|\left\|\left(P_{n} \psi-\psi\right)\right\|
\end{aligned}
$$

For $\psi \in V_{j}$ we have

$$
\begin{aligned}
\left\|H^{1 / 2}\left(P_{n} \psi-\psi\right)\right\|^{2} & =\left\langle\left(P_{n} \psi-\psi\right), H\left(P_{n} \psi-\psi\right)\right\rangle \\
& <C\left\langle\left(P_{n} \psi-\psi\right), A^{2}\left(P_{n} \psi-\psi\right)\right\rangle=C\left\|A\left(P_{n} \psi-\psi\right)\right\|^{2},
\end{aligned}
$$

where $A$ is the Hermite operator whose eigenfunctions are $\left\{\Omega_{j}\right\}$. (A5.7) follows from the observation that $H=A+B$, where $B$ is a fourth degree polynomial, and there exists a constant $C$ so that as forms on $\mathcal{\bigotimes} \bigotimes$

$$
H \leqslant C A^{2} \text {. }
$$

Thus, for some constant $D$,

$$
\begin{aligned}
0 & <E_{j}^{(n)}-E_{j} \\
& <D \max _{\substack{\psi \in V_{j} \\
\|\psi\|=1}}\left[\left\|A\left(P_{n} \psi-\psi\right)\right\|\|A \psi\|+\|H \psi\|\left\|P_{n} \psi-\psi\right\|+\varepsilon_{n}\|A \psi\|^{2}\right] .
\end{aligned}
$$


The right side of (A5.9) tends to zero as $n \rightarrow \infty$ because $\left\|A P_{n} \psi-A \psi\right\|=\| P_{n}(A \psi)$ $-A \psi \|$. To see that the convergence is faster than any power of $n^{-1}$, note that $V_{j}=\operatorname{span}\left\{\psi_{k}\right\}_{k=0}^{j} \subset \delta$ since each $\psi_{j} \in \mathcal{S}[20],[21]$.

Since the Hermite expansion $\psi=\Sigma c_{l} \Omega_{l}$ of a function $\psi \in \delta$ has coefficients which decay faster than any power of $l^{-1}$, (A5.1) follows from (A5.9).

Proof of (A5.2). Note that

$$
\begin{gathered}
\left\|\psi_{j}-\psi_{j}^{(n)}\right\|<\left\|\psi_{j}-P_{n} \psi_{j}\right\|+\left\|P_{n} \psi_{j}-\left\langle\psi_{j}, \psi_{j}^{(n)}\right\rangle \psi_{j}^{(n)}\right\| \\
+\left\|\left\langle\psi_{j}, \psi_{j}^{(n)}\right\rangle \psi_{j}^{(n)}-\psi_{j}^{(n)}\right\| .
\end{gathered}
$$

However, if we choose the phase of $\psi_{j}^{(n)}$ so that $\left\langle\psi_{j}, \psi_{j}^{(n)}\right\rangle>0$, then

$$
\begin{aligned}
\left\|\left\langle\psi_{j}, \psi_{j}^{(n)}\right\rangle \psi_{j}^{(n)}-\psi_{j}^{(n)}\right\| & =1-\left\langle\psi_{j}, \psi_{j}^{(n)}\right\rangle \\
& \leqslant\left(1-\left\langle\psi_{j}, \psi_{j}^{(n)}\right\rangle\right)^{1 / 2}=2^{-1 / 2}\left\|\psi_{j}-\psi_{j}^{(n)}\right\| .
\end{aligned}
$$

Thus,

$$
\left\|\psi_{j}-\psi_{j}^{(n)}\right\|<\left(1-2^{-1 / 2}\right)^{-1}\left[\left\|\psi_{j}-P_{n} \psi_{j}\right\|+\left\|P_{n} \psi_{j}-\left\langle\psi_{j}, \psi_{j}^{(n)}\right\rangle \psi_{j}^{(n)}\right\| .\right.
$$

Therefore, it suffices to show that $\left\|P_{n} \psi_{j}-\left\langle\psi_{j}, \psi_{j}^{(n)}\right\rangle \psi_{j}^{(n)}\right\|$ tends to zero faster than any power of $n^{-1}$. Observe that

$$
\left\|P_{n} \psi_{j}-\left\langle\psi_{j}, \psi_{j}^{(n)}\right\rangle \psi_{j}^{(n)}\right\|^{2}=\sum_{\substack{k=0 \\ k \neq j}}^{n}\left\langle P_{n} \psi_{j}, \psi_{k}^{(n)}\right\rangle^{2} .
$$

We estimate this sum by noting

$$
\begin{aligned}
& \left\langle P_{n} \psi_{j}, H \psi_{k}^{(n)}\right\rangle=\left\langle\psi_{j}, P_{n} H \psi_{k}^{(n)}\right\rangle=E_{k}^{(n)}\left\langle P_{n} \psi_{j}, \psi_{j}^{(n)}\right\rangle, \\
& \left\langle P_{n} H \psi_{j}, \psi_{k}^{(n)}\right\rangle=E_{j}\left\langle P_{n} \psi_{j}, \psi_{k}^{(n)}\right\rangle,
\end{aligned}
$$

so that

$$
\left\langle P_{n} \psi_{j}, \psi_{k}^{(n)}\right\rangle=\left(E_{j}-E_{k}^{(n)}\right)^{-1}\left\langle P_{n} H\left(I-P_{n}\right) \psi_{j}, \psi_{k}^{(n)}\right\rangle .
$$

Since the eigenvalues $E_{j}$ are nondegenerate, and since $E_{k}^{(n)}$ can be made uniformly close to $E_{k}$ (for $k=0,1, \ldots, j+1$ ) when $n$ is sufficiently large (by (A5.1)), there is a $\delta>0$ such that $0<\delta<\left|E_{j}-E_{k}^{(n)}\right|$ for $k \neq j$ when $n$ is sufficiently large.

Hence,

$$
\begin{aligned}
\left\|P_{n} \psi_{j}-\left\langle\psi_{j}, \psi_{j}^{(n)}\right\rangle \psi_{j}^{(n)}\right\|^{2} & \leqslant \delta^{-2}\left\|P_{n} H\left(I-P_{n}\right) \psi_{j}\right\|^{2} \\
& =\delta^{-2} \sum_{k=0}^{n}\left\langle\Omega_{k}, H\left(I-P_{n}\right) \psi_{j}\right\rangle^{2} \\
& =\delta^{-2} \sum_{k=0}^{n}\left(\sum_{l=n+1}^{\infty}\left\langle\Omega_{k}, H \Omega_{l}\right\rangle\left\langle\Omega_{l}, \psi_{j}\right\rangle\right)^{2} .
\end{aligned}
$$

Because of the form of $H$, the last expression is equal to

$$
\delta^{-2} \sum_{k=n-3}^{n}\left(\sum_{l=n+1}^{k+4}\left\langle\Omega_{k}, H \Omega_{l}\right\rangle\left\langle\Omega_{l}, \psi_{j}\right\rangle\right)^{2} .
$$

It follows from (A6.4) that the terms $\left\langle\Omega_{k}, H \Omega_{l}\right\rangle$ are $O\left(n^{2}\right)$ in the appropriate range of $k, l$. Thus, (A5.10) decays faster than any power of $n^{-1}$, because the coefficients $\left\langle\Omega_{l}, \psi_{j}\right\rangle$ decay faster than any power of $l^{-1}$. 
We do not give the proof of convergence for the Ising basis. However, we remark that since finite linear combinations of Ising basis elements $\phi_{j}^{e}$ and $\phi_{j}^{o}$ yield the Hermite functions $\Omega_{j}^{+}$and $\Omega_{j}^{-}$, the eigenvalues found using the Ising basis are squeezed between the true eigenvalues and those of a Hermite operator localized in one of the two wells. A simple modification of the above proof then shows that the eigenvalues found using the Ising basis satisfy (A5.1).

We conjecture that the true rate of convergence is exponential. This would follow if the eigenfunctions of $H$ were analytic vectors of some power of $A$.

Appendix 6. Formulas for Some Matrix Elements. We consider only the Gaussian basis and derive explicit formulas for the elements of the infinite matrix $H$.

Define

$$
\begin{aligned}
X_{j, k}^{n} & \equiv\left\langle\Omega_{j}, x^{n} \Omega_{k}\right\rangle ; \\
P_{j, k}^{n} & \equiv\left\langle\Omega_{j},(-i(d / d x))^{n} \Omega_{k}\right\rangle .
\end{aligned}
$$

Then we have the recursion relations

$$
\begin{aligned}
X_{j, k}^{0} & =P_{j, k}^{0}=\delta_{j, k} \quad(\text { Kronecker delta }), \\
X_{j, k}^{n} & =(2 \alpha)^{-1 / 2}\left[(j+1)^{1 / 2} X_{j+1, k}^{n-1}+j^{1 / 2} X_{j-1, k}^{n-1}\right], \\
P_{j, k}^{n} & =i(\alpha / 2)^{1 / 2}\left[(j+1)^{1 / 2} P_{j+1, k}^{n-1}-j^{1 / 2} P_{j-1, k}^{n-1}\right] .
\end{aligned}
$$

It follows from (2.1) and (A6.1)-(A6.3) that

$$
\begin{aligned}
H_{j, k} \equiv & \left\langle\Omega_{j}, H \Omega_{k}\right\rangle \\
= & (1 / 4)\left[\left(\alpha^{2}+c \alpha^{-2}+(3 / 2) d \alpha^{-4}\right)(2 j+1)+3 d \alpha^{-4} j^{2}+2 e\right] \delta_{j, k} \\
& +(1 / 4)\left(j^{2}-j\right)^{1 / 2}\left[-\alpha^{2}+c \alpha^{-2}+d \alpha^{-4}(2 j-1)\right] \delta_{j, k+2} \\
& +(1 / 4)\left(k^{2}-k\right)^{1 / 2}\left[-\alpha^{2}+c \alpha^{-2}+d \alpha^{-4}(2 k-1)\right] \delta_{j+2, k} \\
& +(1 / 8) d \alpha^{-4}[j(j-1)(j-2)(j-3)]^{1 / 2} \delta_{j, k+4} \\
& +(1 / 8) d \alpha^{-4}[k(k-1)(k-2)(k-3)]^{1 / 2} \delta_{j+4, k}
\end{aligned}
$$

This is a nine-diagonal band matrix which reduces to a pentadiagonal band matrix in each subspace $\mathcal{H}_{e}, \mathcal{K}_{o}$.

Acknowledgements. We would like to thank James Glimm and Eugene Isaacson for their valuable advice, support, and encouragement. We would also like to thank Olof Widlund for several enjoyable and enlightening conversations.

Department of Mathematics

Rensselaer Polytechnic Institute

Troy, New York 12181

Department of Mathematics

Rockefeller University

New York, New York 10021

Department of Mathematics

Pontificia Universidade Catolica do Rio de Janeiro

Rio de Janeiro, Brazil 
Department of Mathematics

Pontificia Universidade Catolica do Rio de Janeiro

Rio de Janeiro, Brazil

1. D. Isancson, D. Marchesin \& P. J. Paes-Leme, Second Internat. Conf. on Computational Methods in Nonlinear Mechanics, T.I.C.O.M., 1979, p. 185; Internat. J. Engrg. Sci., v. 18, 1980, pp. 341-349.

2. N. W. BAzLeY \& D. W. Fox, "Lower bounds for eigenvalues of Schrödinger's equation," Phys. Rev. (2), v. 124, 1961, pp. 483-492.

3. J. L. Richardson \& R. BlankenBeCler, "Moment recursions and the Schrödinger problem," Phys. Rev. D, v. 19 (3), 1979, pp. 496-502.

4. D. IsAncson, "Singular perturbations and asymptotic eigenvalue degeneracy," Comm. Pure Appl. Math., v. 29, 1976, pp. 531-551.

5. D. MArchesin, "The scaling limit of the $\varphi^{2}$ field in the anharmonic oscillator," J. Math. Phys., v. 20, 1979, pp. 830-836.

6. D. IsAAcson, "The critical behavior of $\phi_{1}^{4}$," Comm. Math. Phys., v. 53, 1977, pp. 257-275.

7. A. H. Stroud \& D. Secrest, Gaussian Quadrature Formulas, Prentice-Hall, Englewood Cliffs, N. J., 1966.

8. B. T. SMrth ET AL., Matrix Eigensystem Routines, Eispack Guide, Springer-Verlag, Berlin and New York, 1974.

9. G. TeMPLE, "The theory of Rayleigh's principle as applied to continuous systems," Proc. Roy. Soc. Ser. A, v. 119, 1928, pp. 276-293.

10. T. KATo, Perturbation Theory for Linear Operators, Springer-Verlag, Berlin and New York, 1972.

11. J. H. Winknsson, The Algebraic Eigenvalue Problem, Clarendon Press, Oxford, 1965.

12. J. D. P. Donnelly, "Bounds for the eigenvalues of self-adjoint operators," SIAM J. Numer. Anal., v. 7, 1970, pp. 458-478.

13. P. DirAc, Quantum Mechanics, Clarendon Press, Oxford, 1935.

14. E. M. HARReLL, "On the rate of asymptotic eigenvalue degeneracy," Comm. Math. Phys., v. 60, 1978, pp. 73-95.

15. S. G. Mixhnn, The Numerical Performance of Variational Methods, Wolters-Noordhoff, Groningen, 1971.

16. W. RrTz, "Über eine neue Methode zur Lösunggewisser Variationsprobleme der Mathematischen Physik," J. Reine Angew. Math., v. 135, 1908, pp. 1-61; "Theorie der Transversalschwingungen einer quadratischen Platte mit freien Rändern," Ann. Physik, v. 28, 1909, pp. 737-786.

17. S. G. MrkhIIN, Variationsmethoden der Mathematischen Physik, Akademie-Verlag, Berlin, 1962.

18. M. A. Krasnosel'skii, G. M. VAINIKKo Et AL., Approximate Solution of Operator Equations, Wolters-Noordhoff, Groningen, 1972.

19. G. Strang \& G. Fix, An Analysis of the Finite Element Method, Prentice-Hall, Englewood Cliffs, N. J., 1973.

20. E. C. Trtchmarsh, Eigenfunction Expansions Associated With Second-Order Differential Equations, Vols. 1 and 2, Clarendon Press, Oxford, 1958.

21. A. JAFFE, Dynamics of a Cutoff $\lambda \phi^{4}$ Field Theory, Princeton thesis, 1965. 